\title{
What is a GMC? Are observers and simulators discussing the same star-forming clouds?
}

\author{
Hsi-An Pan ${ }^{1}$, Yusuke Fujimoto ${ }^{1}$, and Elizabeth J. Tasker ${ }^{1}$ \\ ${ }^{1}$ Department of Physics, Hokkaido University, \\ Kita 8, Nishi 5, Kita-ku, Sapporo, Hokkaido 060-0808, Japan \\ email: hapan@astro1.sci.hokudai.ac.jp
}

\begin{abstract}
Observations and simulations have now reached the point where the giant molecular cloud (GMCs) populations can be studied over a whole galaxy. This is immensely helpful for understanding star formation. Yet, are these two groups really comparing the same objects? While simulators work in $6 \mathrm{D}\left(x, y, z, v_{x}, v_{y}, v_{z}\right)$ position-position-position (PPP) space, observers see $2+1 \mathrm{D}$ (RA, Dec, $v_{l o s}$ ) projected properties along the line of sight, identifying clouds in position-position-velocity (PPV) space. In this research we generated PPP and PPV data for a high-resolution simulated galaxy and compared the identified clouds in both data sets. The results show that $70 \%$ of the clouds have a single counterpart in each data structure. Cloud boundaries of these clouds are indeed the same. Scatter of the derived cloud properties (radius and velocity dispersion) between PPP and PPV are typically within a factor of two. However, this small scatter can make it difficult to determine if a cloud is truly gravitationally bound.
\end{abstract}

Keywords. methods: numerical, techniques: image processing, ISM: clouds, ISM: structure, galaxies: ISM

\section{Introduction}

Physical properties of giant molecular clouds (GMCs) - the birthplace of stars - are of vital importance. They reveal clues as to why stars are formed in certain regions, how many stars can form and how star formation proceeds.

Thank to ALMA, we are rapidly reaching the point where simulations and observations will achieve comparable resolution of GMCs in a wide variety of galaxies. This opens the door to truly constrain the mechanisms for cloud formation and star formation. However, this can be challenging since the two techniques are based on different data structures. Simulations have data with 3D position $(x, y, z)$ and velocity $\left(v_{x}, v_{y}, v_{z}\right)$, so called (position-position-position space or PPP), from which GMC properties are directly calculated. On the other hand, observations take data from galaxies projected on the sky plane. This provides two spatial dimensions (RA and Dec) and a velocity along the line of sight, known as the spectral data cube in positionposition-velocity space (PPV).

In this work, we compared the physical properties of GMCs formed in a simulation of a barred spiral galaxy using simulation and observational identification methods. More detail can be found in Pan et al.(2015) MNRAS, 453, 3082.

\section{Cloud Identification}

In both data structures, GMCs are identified as continuous structures of gas above a chosen density or flux threshold. PPP works from the lowest density, drawing a contour at $n_{\mathrm{HI}, \text { thresh }}=$ $100 \mathrm{~cm}^{-3}$ and defining all cells within a closed section as the cloud. This method was originally introduced by Fujimoto et al.(2014). PPV clouds are identified using the CPROPS package developed by Rosolowsky \& Leroy(2006). The package was designed to identify contentious 

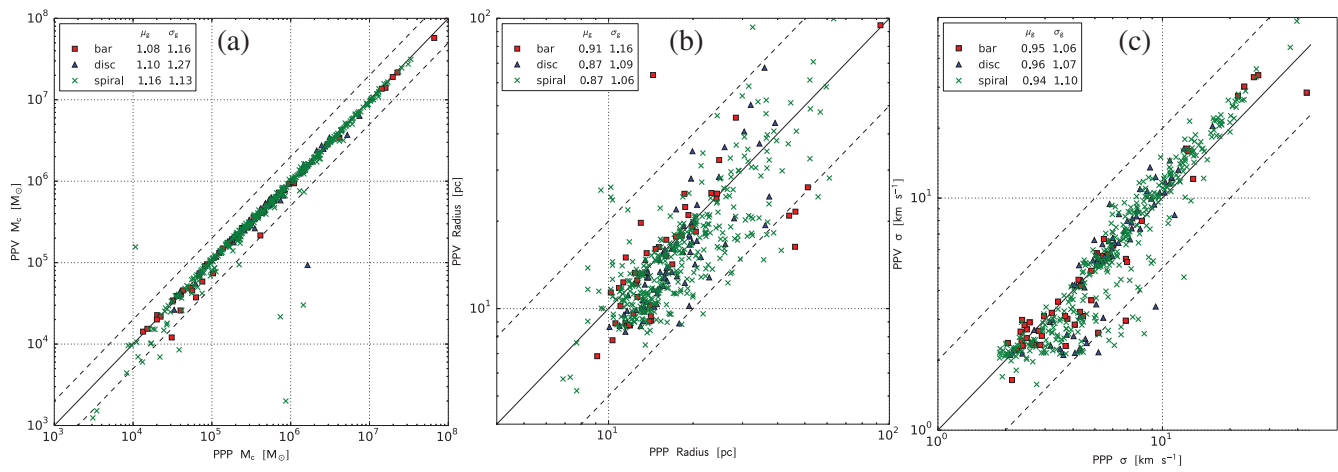

Figure 1. Comparison of match cloud properties. Colour symbols denote clouds in different galactic environments: green crosses are spiral clouds, red squares are bar clouds, and blue triangles show disc clouds. Panel (a) - (d) show the comparison between cloud mass, radius, velocity dispersion, and virial parameter, respectively. Solid line indicate $1: 1$. Dashed lines indicate a factor of 2 above and below the solid line. Geometric mean $\left(\nu_{g}\right)$ and geometric standard deviation $\left(\sigma_{g}\right)$ of the ratio of PPV to PPP properties are provided for galactic environments in each panel.

structures in observations. CPROPS begins by masking the emission with a high signal-to-noise ratio $(\mathrm{S} / \mathrm{N})$, picking out the cloud locations at densities much higher than the background. It then extends this mask to the user defined lowest $\mathrm{S} / \mathrm{N}$, which outlines the observed cloud boundary. Physical properties of the clouds are derived once the cloud boundaries are set. We refer readers to Rosolowsky \& Leroy(2006), Fujimoto et al.(2014) and Pan et al.(2015) for the details.

\section{Results and Implication}

The total number of clouds identified was similar in the PPV and PPP methods (971 versus 1029) in the galaxy. This was also true when comparing cloud numbers in each galactic environment of bar, spiral and disc. The typical (median) cloud properties, such as their mass, radius and velocity dispersion, differ by $<20 \%$ between PPV and PPP.

We further directly compared individual clouds that have been identified in both PPP and PPV space. The cloud matching method is visualized in Pan et al.(2015). Our method suggests that $70 \%$ of the clouds were successfully matched to a single counterpart. The high match rate is attributed to the thin galactic disc which reduces the projection effect. Figure 1 shows the plots of one-to-one cloud properties for the $70 \%$ match clouds, including mass, radius, velocity dispersion, and virial parameter. The first three properties scatter within 2 times between two data. Large scatter is seen in the virial parameter because this property depends on cloud mass, radius, and velocity dispersion. This makes it difficult to tell if a cloud is gravitationally bound in observations, since a spread of a factor of 2 can turn a bound cloud into an unbound object.

\section{References}

Fujimoto, Y., Tasker, E. J., Wakayama M., \& Habe, A. 2014, MNRAS, 439, 936

Pan, H.-A., Fujimoto, Y., Tasker, E. J. et al. 2015, MNRAS, 453, 3082

Rosolowsky, E., \& Leroy, A. 2006, PASP, 118, 590 